Cahiers d'études africaines

\title{
Une analyse du discours international sur la « fuite des cerveaux ». Un consensus en trompe-l'œil
}

An Analysis of International Narratives on the "Brain Drain"

\section{Antonina Levatino et Antoine Pécoud}

\section{(2) OpenEdition}

\section{Journals}

Édition électronique

URL : http://journals.openedition.org/etudesafricaines/17637

DOI : 10.4000/etudesafricaines. 17637

ISSN : 1777-5353

Éditeur

Éditions de l'EHESS

\section{Édition imprimée}

Date de publication : 30 juin 2014

Pagination : 195-215

ISSN : 0008-0055

\section{Référence électronique}

Antonina Levatino et Antoine Pécoud, «Une analyse du discours international sur la « fuite des cerveaux ». Un consensus en trompe-l'œil », Cahiers d'études africaines [En ligne], 213-214 | 2014, mis en ligne le 27 juin 2016, consulté le 10 décembre 2020. URL : http://journals.openedition.org/ etudesafricaines/17637 ; DOI : https://doi.org/10.4000/etudesafricaines.17637

Ce document a été généré automatiquement le 10 décembre 2020.

(c) Cahiers d'Études africaines 


\title{
Une analyse du discours international sur la « fuite des cerveaux ». Un consensus en trompe-l'œil
}

\author{
An Analysis of International Narratives on the "Brain Drain"
}

Antonina Levatino et Antoine Pécoud

1 Depuis une à deux décennies, les migrations internationales font l'objet d'une attention croissante au niveau international. Les organisations internationales (OI), en particulier, se sont emparées de ce thème et ont, entre autres efforts, produit plusieurs rapports dont le but est d'esquisser ce qui pourrait (ou devrait) constituer les orientations principales des politiques migratoires. Un des présupposés de ce type d'initiative est que les migrations constituent un enjeu d'importance mondiale, mais qui n'est pas actuellement reconnu comme tel par les États et la communauté internationale; contrairement à d'autres enjeux "globaux », les migrations seraient encore abordées de façon unilatérale par les gouvernements et ce déficit de coopération internationale nuirait au succès de leurs politiques en la matière. Il s'agit donc d'«internationaliser» les questions migratoires; dans ce processus, l'établissement d'un discours international sur les migrations, qui définit à la fois les questions à poser et les réponses à y apporter, est une étape nécessaire.

2 Cet article examine la façon dont ce discours traite les questions soulevées par les migrations dites «qualifiées ». Cette catégorie de migrants est malaisée à définir, mais englobe généralement les détenteurs d'un diplôme universitaire (ou équivalent) et des professions comme ingénieurs, chercheurs, enseignants, médecins ou infirmières (Betts \& Cerna 2011). Ce type de migration est régulièrement associé à des conséquences négatives, comme la "fuite des cerveaux", et soulève un ensemble de questions économiques, politiques et éthiques concernant, par exemple, le rôle des États dans l'admission et le recrutement des "cerveaux" des pays pauvres; son impact sur le développement et la situation des populations restées au pays (en particulier dans le 
domaine de la santé ou de l'éducation); ou le rôle respectif des marchés et des gouvernements dans des segments mondialisés du marché du travail. La question est donc de comprendre comment les or abordent ces enjeux sensibles et produisent un récit qui surmonte ces dilemmes.

Cet article décrit d'abord les tentatives actuelles visant à améliorer la "gouvernance » des migrations internationales, ainsi que les obstacles auxquels elles font face. Il examine ensuite le rôle des discours dans ce processus, et présente le corpus des rapports sur lequel est basée notre analyse. Il donne un aperçu des débats autour des migrations qualifiées et des arguments qui ont été développés à ce sujet. Dans ce contexte, les or tentent de développer une vision globalement optimiste des migrations, ce qui les conduit à remettre en question les considérations pessimistes sur la "fuite des cerveaux ». Il présente ensuite les recommandations des $\mathrm{OI}$ en la matière et analyse la façon dont ces récits concilient le rôle respectif des États et du secteur privé dans la dynamique des migrations qualifiées.

\section{Vers une « gouvernance mondiale des migrations »?}

Les flux migratoires constituent par définition un processus transnational qui crée des interdépendances entre pays. Cependant, il n'existe que très peu d'instruments ou de mécanismes de régulation des politiques migratoires mises en œuvre par les différents États. Beaucoup d'observateurs ont ainsi souligné l'absence d'une institution internationale sur le sujet. Le mandat du Haut commissariat des Nations Unies pour les réfugiés (HCR) ne concerne que les réfugiés, tandis que l'Organisation internationale pour les migrations (OIM) n'appartient pas au système des Nations Unies; d'autres aspects des migrations (droits des migrants, «traite » ou «trafic » de personnes) sont quant à eux abordés par d'autres agences ${ }^{1}$. Le résultat est un panorama fragmenté qui encourage la compétition et la rivalité entre agences (Betts 2011). De plus, le cadre normatif en la matière est faible : bien que la protection des travailleurs migrants ait déjà figuré dans le mandat de l'ort lors de sa création en 1919 (Rosental 2006), les instruments de droit international en la matière n'ont jamais été ratifiés par un nombre d'États suffisamment élevé pour leur donner une véritable influence (de Guchteneire \& Pécoud 2010). Dans une large mesure, les politiques migratoires restent donc un enjeu de stricte souveraineté des États, que ces derniers traitent de façon essentiellement unilatérale.

La situation est cependant en train d'évoluer. Les migrations firent l'objet de discussions lors de la Conférence internationale sur la population et le développement, organisée au Caire en 1994, et plusieurs initiatives ont été prises depuis 2000 : on peut notamment mentionner les travaux de la Commission mondiale sur les migrations internationales ( CMMI) de 2003 à 2005 ; l'organisation d'un « Dialogue de haut niveau sur les migrations internationales et le développement » à l'oNu en 2006 et en 2013, et du Forum mondial sur la migration et le développement (FMMD), qui se tient annuellement depuis 2007 ; ainsi que d'autres efforts comme l'Initiative de Berne (2001) ou le Processus de la Haye (2000). Au niveau régional, les migrations ont progressivement fait leur apparition sur l'agenda de l'Union européenne, tandis que des processus de consultation régionale ont été créés dans la plupart des autres continents (Channac 2006).

6 Une des principales idées sous-jacentes à ces initiatives est qu'une coordination accrue entre États permettrait aux politiques migratoires de produire de meilleurs résultats. Les 
principaux thèmes dans ces débats sont notamment le lien entre migrations et développement (par le biais des transferts de fonds et du rôle des diasporas en particulier), la lutte contre la «traite» des êtres humains, la protection des migrants et de leurs droits, ainsi que d'autres sujets qui font l'objet d'une attention croissante (comme l'impact du changement climatique sur les déplacements de personnes). Les migrations qualifiées jouent un rôle central dans ces préoccupations, dans la mesure où elles constituent un obstacle potentiel au développement et au respect des droits fondamentaux dans les régions de départ (surtout dans le cas de l'émigration des professionnels de santé et des enseignants).

7 Mais comme le montrent Betts et Cerna (2011), il existe de nombreux obstacles à une approche multilatérale et concertée des migrations qualifiées. Ces dernières ont des conséquences redistributives, car elles tendent à être bénéfiques pour les pays de destination tout en imposant des coûts à ceux d'origine; l'interdépendance entre gouvernements est donc asymétrique. De plus, les États de départ n'ont que peu d'influence sur l'émigration de leurs citoyens qualifiés et sont donc relativement impuissants à réguler ces flux. Par ailleurs (et contrairement aux migrations non qualifiées), les États de destination voient généralement les migrations qualifiées comme souhaitables et, étant donné l'offre limitée de personnel qualifié, tendent à rivaliser pour les attirer, ce qui n'encourage pas la coopération. Il est donc intéressant de voir comment, face à ces déséquilibres et obstacles structurels, les or tentent de développer une approche consensuelle des migrations qualifiées.

\section{Naissance d'un discours international sur les migrations}

8 La "gouvernance " internationale des migrations implique la coopération d'un grand nombre d'acteurs différents, à savoir non seulement les gouvernements des pays concernés, mais aussi la société civile, les ONG, les or ou le secteur privé. Afin de rendre une discussion possible, ces acteurs (ou "parties prenantes» dans le jargon des oI) doivent converger sur un certain nombre de principes communs. Il s'ensuit le besoin de " récits ", qui proposent un cadre interprétatif partagé de l'enjeu en question et des pistes pour agir. Les mécanismes de gouvernance internationale se caractérisent en effet par l'absence de relations formelles d'autorité entre acteurs, et donc par la nécessaire adhésion de ces derniers aux principes communs. Cela ne signifie naturellement pas que tous les participants sont sur un pied d'égalité, mais souligne tout de même le besoin d'idées communes sur ce qu'il convient de faire - d'où les contacts et échanges répétés entre acteurs (qu'illustrent des processus comme le FMMD) et l'existence de discours consensuels sur le sujet (Rosenau \& Czempiel 1992).

9 C'est dans ce contexte qu'on peut comprendre le nombre élevé de rapports internationaux consacrés aux migrations durant cette dernière décennie. Citons par exemple les sept World Migration Reports de l'огм (publiés entre 2000 et 2013), le Rapport sur le développement humain du Programme des Nations Unies pour le développement (PNUD 2009), consacré aux migrations, ainsi que les rapports produits par les initiatives mentionnées plus haut (cMmI, FMMD, Dialogue de haut niveau, Initiative de Berne, Processus de la Haye, etc.). Ce corpus a deux objectifs : présenter ce que "sont» les migrations (volumes, dynamiques, tendances, impact, etc.) et identifier des «bonnes» 
politiques migratoires. Le mandat de la CMMI (2005 : vii) était ainsi « de fournir un cadre pour la formulation d'une réponse cohérente, globale et complète à la question des migrations internationales $»$.

Le discours international sur les migrations élabore donc un cadre analytique et normatif qui, sur le papier du moins, fédère les préoccupations des différents acteurs et transcende les oppositions et les dilemmes qui les divisent. C'est particulièrement clair dans l'objectif dit "gagnant-gagnant-gagnant", selon lequel les migrations devraient bénéficier aux pays de destination et de départ, ainsi qu'aux migrants eux-mêmes (Badie et al. 2008). Ce discours correspond également au mandat des institutions internationales qui est de fournir aux États une "expertise » sur un enjeu donné et des recommandations sur la manière de le traiter politiquement. Cette fonction, qui conjugue capacité de production de savoirs «techniques » et influence normative sur les politiques des États, est une des principales raisons d'être des or (Barnett \& Finnemore 1999).

11 Il est en conséquence important de souligner que ce discours ne fait pas qu'analyser les migrations, ou refléter les points de vue des États ou d'autres acteurs. En l'absence de récits partagés par tous, les or cherchent activement à produire un consensus qui n'existait pas avant leur intervention. Analysant les discours de l'onu sur le développement, Rist (2002: 25) démontre ainsi «comment le discours, qui se prétend référentiel et qui est donc censé décrire la réalité, parvient à construire cette réalité ». Les rapports de notre corpus font des migrations un enjeu «global» et tentent de transformer ce qui a longtemps été perçu comme une question de souveraineté nationale en un objet de gouvernance mondiale (Amaya-Castro 2012).

\section{Le corpus}

Notre corpus se compose d'un échantillon de rapports internationaux, qui reflètent les vues des principales institutions internationales (Banque Mondiale, Organisation mondiale de la santé, Organisation mondiale du commerce, OIT, OIM, PNUD) et d'autres initiatives importantes à ce niveau (сMMI, Processus de la Haye). Ces rapports sont les suivants, par ordre chronologique :

Déclaration de La Haye sur la politique de demain en matière de réfugiés et de migrations (Nations Unies 2002) ; Rapport sur le commerce mondial 2004 (омc 2004); Rapport de la Commission mondiale sur les migrations internationales (CMMI 2005); Rapport «Migrations internationales et développement» de l'oNU (Nations Unies 2006) ; Rapport sur la santé dans le monde 2006 (oms 2006); Cadre multilatéral de l' oIT pour les migrations de main-d'œuvre (ort 2006); Rapport sur le développement dans le monde 2007 (Banque Mondiale 2007) ; Rapport sur le développement humain 2009 (PNUD 2009); Rapport sur le développement dans le monde 2009 (Banque Mondiale 2009) ; État de la migration dans le monde 2010 (OIM 2010).

Notre analyse n'insiste pas sur les différences possibles entre ces rapports, mais les considère plutôt comme un corpus unique au sein duquel les divergences comptent moins que les convergences. Cela peut sembler étonnant, dans la mesure où les observateurs soulignent régulièrement les différences d'approche entre institutions internationales; par exemple, la perspective du PNUD, et sa notion de « développement humain » inspirée par Amartya Sen (1999), s'opposerait explicitement au plus orthodoxe «consensus de Washington » sous-jacent aux projets de la Banque Mondiale. 
Cependant, malgré les incontestables divergences intellectuelles et idéologiques entre ces approches, la manière dont ces or traitent des migrations qualifiées reste assez homogène. Cela rappelle le « compromis » dont parle Utting (2006), qui inspire ce qu'on qualifie généralement de consensus "post-Washington » et qui voit les organisations " néolibérales " (comme celles dites « de Bretton Woods ») incorporer des préoccupations " sociales » et " politiques» (comme le rôle du capital social et des institutions, le genre ou la notion d'empowerment), tandis que les institutions dont le mandat concerne les droits humains et la protection (OIT, $\mathrm{HCDH}$ ) cessent de remettre en cause le modèle social basé sur l'économie de marché et la mondialisation libérale (tout en continuant de critiquer certaines de ses conséquences, en termes de protection du travail, d'inégalités ou de violations des droits fondamentaux).

\section{Le débat sur la « fuite des cerveaux »}

16 La notion de « fuite des cerveaux » est apparue pour la première fois en Grande-Bretagne en 1963, pour désigner l'émigration des scientifiques britanniques vers l'Amérique du Nord. L'expression a ensuite connu une popularité croissante et s'est appliquée aux migrations de personnel qualifié des pays du «Sud» vers ceux du «Nord». Elle représente une interprétation pessimiste des migrations qualifiées dont la conséquence serait de priver les États de départ des ressources humaines nécessaires à leur développement, et donc de perpétuer (voire d'accentuer) les écarts de richesse entre pays. Les principaux utilisateurs de cette notion sont les gouvernements des pays d'origine, ainsi que certains chercheurs et experts, qui dénoncent le coût de la fuite des cerveaux et proposent des compensations possibles comme la « taxe Bhagwati $»^{2}$.

H. de Haas (2010) note que les débats sur les rapports entre migrations et développement s'insèrent de façon plus large dans les changements paradigmatiques au sein des sciences sociales. De ce point de vue, la perception négative des migrations qualifiées correspond à un paradigme structuraliste/marxiste, ainsi qu'à d'autres courants de pensée, comme la théorie de la dépendance (selon laquelle le contact avec le capitalisme occidental alimente le sous-développement au lieu de le combattre) ou le concept de «systèmemonde » de Wallerstein (2006) (qui insiste sur les déséquilibres dans les relations entre économies développées et sous-développées). Cette interprétation pessimiste fut remise en cause au début des années 1990. L'insistance fut alors placée sur le bénéfice potentiel des migrations qualifiées pour les pays de départ, par le biais des transferts de fonds, du rôle des diasporas dans le développement politique et économique de leur pays d'origine, du retour des migrants et des migrations de type "circulaire ", et de l'impact positif des options d'émigration sur la propension des populations à se former (Meyer 2008).

Les notions de " circulation » et de " gain » des cerveaux (brain gain plutôt que brain drain) firent alors leur apparition. Cette interprétation optimiste s'accompagne de l'usage de plus en plus fréquent des termes "mobilité" ou "circulation», jugés plus «dynamiques» que «migration»; cette «mobilité» serait alors une caractéristique inhérente à la mondialisation, qu'il ne convient pas de remettre en cause mais plutôt d'organiser. De surcroit, cette approche s'insère dans certains débats au sein des sciences sociales, notamment autour de la notion d'agency et de la capacité des "pauvres » à développer leurs propres stratégies : dans cette perspective, les migrants ne sont plus les victimes de dynamiques structurelles défavorables, mais des acteurs à part entière; 
l'émigration n'est pas une fuite hors de la pauvreté, mais un élément dans les stratégies des ménages pour améliorer leur sort (Stark 1991).

L'interprétation pessimiste n'a cependant pas disparu. Ces différents paradigmes ne se suivent pas chronologiquement, mais coexistent au sein des débats scientifiques et politiques. La plupart des ONG (Oxfam 2007; Physicians for Human Rights 2004), en particulier, soulignent systématiquement les conséquences négatives des migrations qualifiées. Si les or leur font parfois écho, elles n'en ont pas moins joué un rôle important dans la diffusion des idées optimistes, qui sont en effet compatibles avec leur objectif "gagnant-gagnant-gagnant». Les sections suivantes tentent de cerner de façon plus précise la manière dont elles développent leurs arguments dans ce domaine.

\section{Une approche positive des migrations}

D'une façon générale, les or adoptent une attitude positive à l'égard des flux migratoires. Cette observation de l'ort (2006: 3) est par exemple représentative de la tonalité de la plupart des rapports de notre corpus :

«Les migrations de main-d'œuvre peuvent avoir de nombreux éléments bénéfiques pour les pays qui envoient et reçoivent des travailleurs migrants, ainsi que pour les travailleurs eux-mêmes. Elles peuvent aider tant les pays d'origine que les pays de destination dans leur croissance et leur développement économiques. »

21 Cette interprétation positive s'applique à la fois aux migrations qualifiées et non qualifiées :

«La distinction traditionnelle entre travailleurs qualifiés et non qualifiés est à certains égards sans utilité parce qu'elle ne reflète pas la complexité des migrations internationales. Par exemple, de nombreux pays sont actuellement très intéressés à recruter des migrants spécialistes en technologies et ingénierie de l'information, mais également à attirer des migrants capables de prodiguer des soins de qualité aux personnes âgées et aux enfants. Même s'ils ont des niveaux d'instruction différents, tous ces migrants pourraient être décrits comme des travailleurs essentiels à la société ».(CMMI $2005: 7$ )

Pour les pays développés, les migrations contribuent à « relever les défis économiques et sociaux que représentent le vieillissement et la diminution de leur population » (ibid. : 17). Pour les régions de départ, les migrations "généreraient d'énormes bénéfices, sous la forme de transferts de fonds plus élevés, d'investissements des diasporas et de transfert de connaissances » (ibid.). Et pour les migrants, « la mobilité humaine peut être un moyen extrêmement efficace d'offrir à quelqu'un de bien meilleures perspectives en termes de revenus, de santé et d'éducation " (PNUD 2009: 1). De plus, " pouvoir choisir son lieu de vie est un élément essentiel de la liberté humaine » (ibid.). En toute logique, ces rapports sont donc favorables à davantage de migrations; le PNUD (ibid.) invite par exemple les gouvernements «à moins limiter les déplacements à travers et à l'intérieur de leurs frontières ».

23 Si cette approche positive des migrations semble aller à contre-courant des représentations dominantes dans beaucoup de pays, il faut noter que ces rapports n'envisagent jamais un scénario d'ouverture des frontières ou de liberté de circulation. Ils introduisent au contraire un certain nombre de conditions normatives à respecter; les migrations peuvent être bénéfiques, mais seulement si elles sont "gérées» ou «contrôlées» de façon appropriée: «les migrations contrôlées offrent d'énormes 
possibilités " (Nations Unies 2002: 3). La tâche des or serait alors d'informer (ou d'instruire) les gouvernements sur la «bonne » manière d'aborder les migrations. La notion de "gestion des migrations » (" migration management ») est ici centrale et véhicule une approche « managériale » des flux migratoires (Geiger \& Pécoud 2010). Une des prémices qui justifient cette appréciation positive des migrations est que les États de destination et de départ seraient dans une situation de complémentarité : «Les premiers voient se réduire leur population en âge de travailler tandis que les seconds en ont plus qu'il ne leur en faut» (CMMI 2005 : 16). Cela créerait une convergence d'intérêts en faveur d'une migration "régulée» des pays pauvres vers les pays riches. Mais ce schéma ne fonctionne pas aussi clairement dans le cas des migrations qualifiées, dans la mesure où les pays de départ manquent de personnel dans des secteurs comme la santé ou l'éducation. La situation des systèmes de santé dans certains pays d'émigration est ainsi jugée "particulièrement préoccupante " (Nations Unies 2006 : 67), notamment en raison de l'absence de personnel qualifié qui «a sérieusement compromis la fourniture des soins de santé aux populations locales» (сммі $2005: 26)$. L'oms (2006: 101) parle même des migrations de personnel de santé comme d'une "saignée mortelle ", et ajoute que "lorsqu'un pays est doté d'un système de santé fragilisé, les pertes de personnel peuvent conduire le système tout entier au bord de l'effondrement et les conséquences se mesurent alors en vies humaines perdues ".

Mais ces mêmes rapports n'en considèrent pas moins que les migrations qualifiées sont une réalité déjà incontestable et inévitable. "Les pays industrialisés [...] sont actuellement confrontés à des pénuries de personnel dans des secteurs économiques de haute importance et basés sur le savoir, tels que la santé, l'éducation et les technologies de l'information"; "n'arrivant pas à recruter, former ou retenir dans leur pays le personnel qui leur est nécessaire », ils "se tournent vers le marché du travail mondial pour répondre à leurs besoins de ressources humaines " (cMMI $2005: 14$ ). En d'autres termes, les migrations qualifiées seraient une caractéristique normale au sein de configurations migratoires globalement bénéfiques, tout en remettant en cause le modèle du gagnant-gagnant-gagnant.

\section{Du pessimisme à l'optimisme}

Le résultat de cette ambivalence est que les rapports reconnaissent les interprétations pessimistes des migrations qualifiées, mais les remettent immédiatement en cause. On assiste donc à la coexistence de différents points de vue au sein des mêmes documents. Par exemple, selon l'onU (Nations Unies 2006 : 16) :

«Les petites économies nationales sont très exposées au risque de "fuite des cerveaux", en particulier dans certains secteurs essentiels tels que la santé et l'éducation. C'est ainsi qu'entre 50 et $80 \%$ de l'ensemble des citoyens ayant un niveau d'instruction supérieur de plusieurs petits pays d'Afrique et des Caraïbes vivent à l'étranger. Ces pays ont besoin qu'on les aide à former suffisamment de travailleurs qualifiés mais aussi à retenir ces travailleurs dans le pays, les charges de travail écrasantes, le manque de fournitures et de matériel requis, l'absence de perspectives de carrière, l'isolement professionnel et les rémunérations insuffisantes incitant le personnel qualifié à émigrer. »

Mais quelques lignes plus bas, on peut lire que :

«La migration des personnes qualifiées présente un certain nombre d'avantages : à l'étranger, les migrants ont la possibilité d'acquérir des compétences et une 
expérience ou de compléter celles qu'ils possèdent déjà, et même s'ils restent dans le pays d'accueil, ils peuvent constituer de précieuses ressources pour leur pays d'origine, en tant qu'investisseurs, que philanthropes, détenteurs de connaissances nouvelles ou encore promoteurs du commerce et des échanges culturels ».(ibid.)

Le même raisonnement est à l'œuvre dans la Déclaration de La Haye :

«La mondialisation ajoute des dimensions nouvelles au marché du travail, notamment la demande de travailleurs hautement qualifiés, laquelle aggrave le problème de la fuite des cerveaux des pays pauvres vers les pays riches ».(ibid. $2002: 14)$

Mais :

«[Les migrants qualifiés] envoient de l'argent à leur famille [...]. L'emploi dans les pays de destination génère des envois de fonds atteignant des milliards de dollars par an, soit beaucoup plus que l'aide publique au développement. Les migrants qualifiés qui se sont installés dans d'autres pays, en particulier dans les économies les plus avancées, doivent être encouragés à partager leurs compétences et autres ressources aux fins du développement de leur pays d'origine. Il existe de nombreux exemples de coopération réussie et de réseaux de migrants, par exemple des scientifiques et des techniciens, œuvrant dans l'intérêt des pays qu'ils ont quittés ». (ibid. : 14-15)

Selon van Dijk (2006), un discours est plus convaincant s’il établit un cadre moral partagé avec ses destinataires. Un message serait difficile à faire passer s'il contredit frontalement les valeurs ou les convictions du public. Il en découle une stratégie discursive qui va d'abord dans le sens de ce que l'audience attend, avant d'introduire des idées nouvelles ou différentes. De ce point de vue, les exemples ci-dessus illustrent la manière dont les arguments pessimistes constituent le message initial: les rapports commencent par reconnaître le problème et regretter les pertes causées par la «fuite des cerveaux »; selon un procédé rhétorique de type captatio benevolentiae, cela établit un cadre politique et idéologique basé sur le développement, les droits humains ou la justice dans les rapports Nord-Sud - ce qui correspond vraisemblablement à ce que certains destinataires attendent de ces rapports. C'est seulement dans une seconde étape que les rapports introduisent de «nouvelles » approches centrées sur les transferts de fonds ou le rôle des diasporas. L'argument de la «fuite des cerveaux » joue à cet égard un rôle tactique qui permet la propagation d'idées plus favorables aux migrations qualifiées. L'examen des recommandations émises par ces rapports confirme cette hypothèse.

\section{Entre « recrutement éthique » et « circulation migratoire »}

Lorsqu'il s'agit de proposer des pistes d'action, les rapports identifient deux orientations politiques principales. La première concerne ce qui est souvent qualifié de « recrutement éthique "; elle aspire à intervenir sur le volume et la nature des flux migratoires, afin de les rendre moins nocifs pour les régions de départ. La seconde se concentre sur la notion de «circulation migratoire» et ambitionne d'éviter la perte de personnel qualifié occasionnée par les migrations permanentes et de promouvoir la contribution des migrants au développement de leur pays d'origine. La première orientation est mentionnée, mais contestée, tandis que la seconde fait l'objet d'une unanimité presque totale. 
32 L'oIt (2006 : 34), par exemple, appelle à « adopter des mesures pour atténuer la perte de travailleurs dotés de compétences essentielles, notamment en établissant des lignes directrices concernant le recrutement éthique ». L'ONU (Nations Unies 2006 : 22) demande de même aux " pays à revenu élevé » de "se retenir - ou d'empêcher les agences de recrutement qui travaillent pour eux - de chercher activement à recruter du personnel qualifié dans les pays où sévit déjà une pénurie de personnes qualifiées ", ainsi que « d'appuyer la constitution d'un capital humain dans ces pays » (ibid.: 69). La même observation est faite par l'oms (2006: 104) :

«Les pays d'accueil ont la responsabilité de veiller à ce que ceux qui sont chargés de recruter des travailleurs dans des pays connaissant une grave pénurie de maind'œuvre soient sensibilisés aux conséquences négatives que ce type de recrutement peut avoir. Les futurs employeurs et les agences de recrutement doivent prendre davantage en considération les investissements importants consentis par les pays d'émigration pour former leur personnel soignant ainsi que les conséquences directes de l'émigration de ce personnel. Comme on le fait dans le cadre des accords bilatéraux, des discussions et des négociations avec les ministères de la santé, les services de planification de la main-d'œuvre et les établissements de formation devraient permettre d'éviter les allégations de "débauchage" et d'autres comportements peu scrupuleux en matière de recrutement. »

Cette approche "éthique » a motivé l'adoption par l'oms, en 2010, d'un Code pour le recrutement international des personnels de santé, un traité non contraignant qui fait suite à d'autres du même genre, à l'initiative de gouvernements, d'institutions internationales ou de corporations ${ }^{3}$. Mais ces outils normatifs sont délicats à adopter et à mettre en œuvre, car ils vont à l'encontre des intérêts de certains États-membres de l'oms (Merçay 2014). De plus, ces appels au recrutement éthique contredisent la valorisation des migrations décrite ci-dessus et ne font donc pas l'unanimité. La CMMI (2005:27) soutient ainsi que l'idée de recrutement éthique «ne respecterait pas les principes des droits de l'homme ", " irait à l'encontre de la mondialisation du marché du travail » et «serait de toute façon fort difficile à mettre en pratique ». De plus, « il est douteux que les codes de conduite que certains pays de destination ont formulés pour tenter d'introduire un certain degré d'autorégulation dans le recrutement de professionnels étrangers soient efficaces » (ibid.). La CMMI (ibid.: 21) plaide même pour davantage de migrations qualifiées, à des fins de compétitivité et de croissance : "Gouvernements et employeurs devraient examiner conjointement les obstacles actuels à la mobilité du personnel hautement qualifié, en vue de supprimer ceux qui entravent inutilement la compétitivité économique. "

Notre corpus mentionne également d'autres manières de prévenir ou de compenser l'impact négatif des migrations qualifiées, mais uniquement afin de les rejeter. C'est le cas des stratégies de type «taxe Bhagwati »: selon la CMMI (ibid.: 27), «les propositions appelant les pays qui recrutent des professionnels étrangers à offrir des compensations financières directes aux pays d'origine ne sont pas réalistes ». Les tentatives de prévenir la mobilité des migrants qualifiés sont également rejetées : "La Commission se méfie de solutions expéditives qui chercheraient à empêcher les professionnels hautement qualifiés de quitter leur pays pour chercher de l'emploi ailleurs » (ibid.); en effet, " faire porter la responsabilité de la fuite des travailleurs qualifiés aux travailleurs eux-mêmes est un contresens ; limiter leur mobilité est probablement contre-productif - et bafoue qui plus est le droit fondamental de chacun de quitter son propre pays » (PNUD 2009 : 4). 

des migrants. La Banque Mondiale rejette l'opposition entre «fuite» et "gain» des cerveaux, au profit de cette idée de "circulation »4. La CMMI (2005: 34) souligne que «l'ancien paradigme de l'établissement permanent des migrants fait place progressivement à des migrations temporaires et circulaires ", et recommande aussi la mise en place de politiques favorisant la « circulation » :

«De nos jours, il faut valoriser la croissance de la mobilité des personnes en promouvant l'idée de "circulation des cerveaux", selon laquelle les migrants retournent régulièrement ou occasionnellement dans leur pays et partagent les bénéfices des compétences et des ressources qu'ils ont acquises en vivant et travaillant à l'étranger. Comme il a été recommandé aussi au chapitre précédent, les pays de destination peuvent encourager la migration circulaire en établissant des dispositifs qui facilitent les déplacements des migrants entre leurs pays d'origine et de destination."

Lencept de "fuite des cerveaux » serait donc inapproprié, obsolète, ou "dépassé »: "La notion d'"exode des cerveaux" est quelque peu dépassée car elle implique qu'un migrant quittant son pays n'y retournera pas » (ibid.). L'idée de «circulation » est également associée au progrès technologique qui faciliterait la communication et les déplacements entre États; on perçoit là une manifestation du lien ancien, mis en évidence par A. Mattelart (2009), entre progrès technique et social et de la manière dont les avancées dans le domaine des transports et de la communication ont toujours fait naître, par les perspectives de rapprochement entre individus et régions qu'elles ouvrent, des espoirs de démocratie et de justice.

L'opposition entre « recrutement éthique » et « circulation » reflète en partie celle entre interprétations pessimiste et optimiste. La première reconnaît l'existence de dommages causés par les migrations qualifiées et tente de compenser en intervenant sur les flux migratoires, tandis que la seconde part du principe qu'un scénario sans dommage est possible et cherche à identifier les dynamiques migratoires qui le réaliseraient. On peut sans doute reprocher à la seconde approche de prendre ses désirs pour des réalités; les citations ci-dessus sont en effet basées sur un mélange d'évidence empirique et de changements désirés: le rejet de l'interprétation pessimiste est en partie basé sur l'observation selon laquelle les migrants circulent, mais aussi - voire surtout - sur le souhait de les voir circuler.

Dans une analyse des discours internationaux sur le développement, D. Eade (2007) observe que ces derniers sont à la fois concrets et ambitieux; ils s'enracinent dans la réalité et dans ses problèmes, tout en envisageant un avenir qui serait radicalement différent du temps présent. Notre corpus reconnaît de même l'existence de problèmes réels mais, plutôt que de les affronter directement, préfère développer l'idée d'un monde dans lequel les migrants circuleraient d'une façon qui serait bénéfique pour tous.

\section{États et secteur privé}

39 Les appels en faveur de la " circulation » des migrants ne sont pas propres aux migrations qualifiées, mais constituent actuellement une priorité affichée par de nombreux gouvernements pour toutes les formes de migration de travail (Castles 2006). Selon l'oNU (Nations Unies 2006 : 20) :

Cahiers d'études africaines, 213-214 | 2014 
«Dans le cadre de ces programmes, les migrants bénéficient d'un statut légal et les pays d'origine profitent des envois de fonds et du retour final des migrants, à condition que l'expérience qu'ils ont acquise à l'étranger puisse s'avérer productive dans leur propre pays. Les pays d'accueil obtiennent les travailleurs dont ils ont besoin et ils peuvent multiplier les bénéfices tirés de la migration en permettant aux migrants de séjourner suffisamment longtemps pour épargner. » programmes permettraient en effet également aux États de destination d'éviter des " coûts supplémentaires en termes d'infrastructures et d'intégration socioculturelle » ( OMC 2004 : xviii).

41 Une critique récurrente de cette approche est qu'elle sert davantage les intérêts des employeurs que ceux des migrants (Wickramasekara 2011). Notre corpus se montre en effet très concerné par le rôle du secteur privé et de son rôle dans la "gestion » des migrations qualifiées. Cela l'amène à aborder la question potentiellement délicate des rapports entre gouvernements et employeurs et des divergences possibles entre leurs intérêts respectifs. Consciente de cet enjeu, la CMMI (2005: 3) écrit ainsi que si les politiques migratoires sont «traditionnellement considérées comme étant du ressort des États souverains », il n'en faut pas moins tenir compte du secteur privé :

«Le personnel hautement qualifié apporte une contribution importante à la compétitivité des entreprises et à l'expansion de l'économie mondiale, de sorte qu'il est nécessaire de faciliter sa mobilité. C'est un souci légitime des États de protéger leurs citoyens contre la concurrence déloyale d'étrangers et il est évident qu'ils continueront à agir sur cette base. Cela étant, les gouvernements et le secteur privé devraient examiner conjointement les entraves existantes à la mobilité professionnelle, en vue d'éliminer celles qui empêchent les entreprises de déployer les bonnes personnes au bon endroit et au bon moment ».(ibid. : 22)

Les aspirations des employeurs - « déployer les bonnes personnes au bon endroit et au bon moment»- caractériseraient un secteur privé présenté comme dynamique et entreprenant, mais frustré par des gouvernements hésitants et incapables de réagir suffisamment vite aux besoins de main-d'œuvre :

«Les entreprises privées ont depuis longtemps reconnu l'importance $\mathrm{du}$ développement et du déploiement de talents du monde entier. Cependant, les décideurs politiques au sein des gouvernements ont dû tenir compte d'autres priorités et ont eu tendance à adopter une attitude ambiguë envers les mouvements du personnel hautement qualifié ».(ibid. : 21)

Le secteur privé serait ainsi "de plus en plus soucieux" (ibid.: 10) et appellerait de ses vœux « une attitude plus libérale face au travail migrant international » (ibid.: 17) afin de surmonter « les obstacles actuels à la mobilité du personnel hautement qualifié » (ibid. : 21). G. Boucher (2008) dénonce ce parti-pris néolibéral qui considère comme acquis un contexte capitaliste, lequel est précisément à l'origine des déséquilibres sous-jacents aux dynamiques migratoires. Cette critique est justifiée, mais sous-estime néanmoins l'obsession de la «régulation» qui traverse notre corpus et n'est pas totalement compatible avec le laissez-faire néolibéral ; selon la Déclaration de la Haye (Nations Unies 2002 : 11) par exemple, « une approche exhaustive et planifiée est nécessaire pour gérer les flux migratoires ». De même, le terme (ou l'idéal) de migrations " ordonnées » est une constante: «la migration humaine et ordonnée est bénéfique pour les migrants et la société » (OIM $2010: 4$ ). Cette tension entre l'ordre étatique et la fluidité d'un marché du travail libéralisé fait souhaiter à la CMMI (2005 : 18) une «libéralisation bien régulée du marché mondial du travail ». 

de recrutement dans les flux de migrants qualifiés. L'onU (Nations Unies 2006 : 69-70) souligne «la multiplication des agences privées de recrutement spécialisées dans le placement de travailleurs qualifiés dans les pays développés ", qui remettrait en question le rôle des États dans l'admission de travailleurs migrants. Cela serait préoccupant car «certaines personnes et certaines entreprises intervenant dans le processus de recrutement trompent et escroquent les migrants qu'ils embauchent, généralement en leur donnant des informations erronées, de fausses promesses et de faux espoirs » (смMI 2005 : 76). La plupart des rapports soulignent donc le besoin « d'une législation et des politiques comportant des mécanismes d'application et des sanctions efficaces pour dissuader l'emploi de pratiques contraires à l'éthique, y compris des dispositions concernant l'interdiction pour les agences d'emploi privées d'utiliser de telles pratiques et la suspension ou le retrait de leur licence en cas de violation » (огт $2006: 29$ ).

On retrouve dans ces citations certaines dynamiques discursives à l'œuvre dans les récits sur la «traite» des êtres humains et l'immigration irrégulière, qui accusent les « passeurs » sans jamais mentionner la responsabilité des États dans la mise en place d'un contexte propice à ces abus (Berman 2010). Cette rhétorique souligne indirectement les limites de ces rapports qui parlent aux gouvernements tout en sachant que ces derniers ne sont pas les seuls acteurs dans l'organisation des flux migratoires. Une approche purement libérale consisterait à donner aux employeurs le pouvoir de recruter qui bon leur semble (et donc à militer pour une certaine forme d'ouverture des frontières), tandis que les recommandations destinées aux États impliquent une insistance sur l'ordre et la régulation. Le discours international sur les migrations navigue entre ces deux scénarios. qualifiées : dans les pays de départ, elle permet la création d'une force de travail qualifiée qui est ensuite susceptible d'émigrer tandis que, dans les pays de destination, elle peut ne pas parvenir à former assez de personnel, ce qui crée une demande de migrants qualifiés. Dans notre corpus, l'éducation est régulièrement présentée comme un investissement nécessaire à la croissance et à la prospérité d'un pays. La CMMI (2005 : 26) écrit que « tous les États devraient consacrer des investissements substantiels à l'éducation et à la formation de leurs citoyens afin d'accroître la compétitivité de leur économie ». Mais la plupart des rapports reconnaissent que cela est non seulement couteux mais aussi risqué pour les pays moins développés, car les personnes qualifiées sont plus à même de trouver des opportunités professionnelles à l'étranger.

Une solution serait que les particuliers paient pour leur propre éducation. La Banque Mondiale y voit la solution à ce dilemme ${ }^{5}$, tandis que le PNUD (2009: 125) parle d'une "réforme du financement de l'éducation", afin "d'élargir l'offre du secteur privé de manière à ce que les personnes désireuses de suivre des formations spécialisées afin de partir à l'étranger ne dépendent pas d'un financement public ». L'éducation est alors un investissement fait par les migrants potentiels, qui peuvent vendre leurs compétences à l'international et accroître ainsi leurs chances de partir à l'étranger. Une autre solution consiste à utiliser l'éducation comme un outil destiné à éviter l'émigration; il s'agit alors de la «formater » afin qu'elle débouche sur des compétences « recherchées dans le pays d'origine, mais moins négociables à l'étranger», ce que le PNUD (ibid.) qualifie de «formation personnalisée pour l'acquisition de compétences ». L'omS (2006:102) parle de même d'« adapter » l'éducation, car « une formation centrée sur la situation locale peut contribuer à limiter l'usure des effectifs $»^{6}$. Comme le note S. Dumitru (2009), on est là en 
présence d'une conception tactique et utilitariste de l'éducation et non d'une représentation de celle-ci comme un droit. ce type de discours. Son objectif principal n'est peut-être pas de construire un argument robuste et cohérent qui s'opposerait à d'autres positionnements intellectuels et idéologiques, mais de transcender tous les désaccords possibles afin d'élaborer un récit qui ne souffre aucune contestation. Ces récits évoquent ce que D. Maingueneau (2002) appelle des discours « constituants » qui ne se réfèrent à rien d'autre qu'à eux-mêmes et aspirent à produire une " vérité » inséparable de l'autorité de ceux qui les produisent. Les rapports de notre corpus sont donc autonomes et se légitiment eux-mêmes. C'est ce qui leur permet d'accueillir des formes acceptées de « double-pensée » (comme Orwell [1950] nomme, dans 1984, l'acceptation simultanée de deux arguments mutuellement incompatibles). consensus dont les or ont besoin. Dans une étude des discours et des pratiques de l'Organisation des Nations Unies pour l'alimentation et l'agriculture (FAO), B. Müller (2009: 27) note que cette institution « entretient un semblant d'harmonie et de consensus là ou existe en fait une large gamme de points de vue et de conflits féroces ». Dans un monde où les intérêts des États sont violemment antagonistes, ces discours peuvent en 
effet représenter l'unique endroit où ces contradictions peuvent être résolues. Mais l'inévitable coût politique de cette stratégie est que les or ne peuvent jamais blâmer qui que ce soit pour les conséquences des migrations qualifiées, au contraire des oNG notamment, qui nomment régulièrement ceux qu'elles considèrent comme " coupables " (à savoir les États occidentaux, les multinationales ou les institutions de Bretton Woods). Les rapports de notre corpus rejettent cette vision du monde marquée par des antagonismes et des rapports de force entre États. Cela les conduit à une représentation naturalisée, et souvent fataliste, de la réalité, selon laquelle l'état du monde n'est pas le produit de déséquilibres ou de conflits, mais de processus vaguement inévitables comme la « mondialisation ». Le résultat final est un consensus en trompe-l'œil, qui n'existe que dans la représentation du monde développée par les oI, et qui entretient une vision dépolitisée des politiques migratoires.

\section{BIBLIOGRAPHIE}

AMAYA-CASTRO, J. M., 2012, « Migration and the World of Work : Discursive Constructions of the Global in ILO Narratives about Migration », in M. GEIGER \& A. PÉCOUd (eds.), The New Politics of International Mobility, Migration Management and its Discontents, Osnabrück, IMIS-Beiträge, 40 : $33-48$.

BADIE, B., BRAuman, R., DeCAuX, E., DeVin, G. \& Wihtol De Wenden, C., 2008, Pour un autre regard sur les migrations, Paris, La Découverte.

BANQUE MONDIALE, 2007, World Development Report 2007 : Development and the Next Generation, Washington, Banque Mondiale.

BANQUE MONDIALE, 2009, World Development Report 2009 : Reshaping Economic Geography, Washington, Banque Mondiale.

BARnetT, M. N. \& FinNemore, M., 1999, « The Politics, Power, and Pathologies of International Organizations », International Organization, 53 (4) : 699-732.

BERMAN, J., 2010, « Réthorique politique, économie rationnelle et trafic des "blanches" à l'ère de la gestion biopolitique », in A. MORICE \& S. РОTOT (dir.), De l'ouvrier immigré au travailleur sans papiers, Paris, Karthala : 95-118.

BETTS, A. (ED.), 2011, « Introduction : Global Migration Governance », in A. BETTS (ed.), Global Migration Governance, Oxford, Oxford University Press : 1-33.

BETTS, A. \& CERNA, L., 2011, « High-Skilled Labour Migration », in A. BETTS (ed.), op. cit. : 60-77. BhagWati, J. N. \& Dellafar, W., 1973, « The Brain Drain and Income Taxation », World Development, $1(1-2): 94-101$.

BOUCHER, G., 2008, «A Critique of Global Policy Discourses on Managing International Migration », Third World Quaterly, 29 (7) :1461-1471.

CASTLES, A., 2006, « Guestworkers in Europe : A Resurrection? », International Migration Review, $40(4): 741-766$. 
CHANNAC, F., 2006, « Vers une politique publique internationale des migrations? ", Revue française de science politique, 56 (3) : 393-408.

COMMISSION MONDIALE SUR LES MIGRATIONS INTERNATIONALES (CMMI), 2005, Les migrations dans un monde interconnecté : nouvelles perspectives d'action, Genève, CMMI.

CRUSH, J., 1995, Power of Development, London, Routlegde.

VAN DIJK, T., 2006, « Discourse and Manipulation », Discourse and Society, 17 (2) : 359-383.

DUMITRU, S., 2009, « L'éthique du débat sur la fuite des cerveaux », Revue européenne des migrations internationales, 25 (1) : 119-135.

EADE, D., 2007, « Editorial », Development in Practice, 17 (4-5) : 467-470.

GEIGER, M. \& PÉCOUD, A., 2010, The Politics of International Migration Management, Basingstoke, Palgrave.

DE GUCHTENEIRE, P. \& PÉCOUD, A., 2010, « Les obstacles à la ratification de la Convention des Nations Unies sur la protection des droits des travailleurs migrants ", Droit et société, 7 : 431-451.

DE HAAS, H., 2010, « Migration and Development : a Theoretical Perspective », International Migration Review, 44 (1) : 1-38.

HoffmanN, S., 1995, « The Crisis of Liberal Internationalism », Foreign Policy, 98 : 159-177.

MAINGUENEAU, D., 2002, « Les rapports des organisations internationales : un discours constituant ? ", in G. RIST (dir.), Les mots du pouvoir. Sens et non-sens de la rhétorique internationale, Paris-Genève, PUF : 119-132.

MATTELART, A., 2009, Histoire de l'utopie planétaire. De la cité prophétique à la société globale, Paris, La Découverte.

MERÇAY, C., 2014 [à paraître] - « Managing the International Migration of Health Workers. The Development of the WHO Code of Practice », Journal of Ethnic and Migration Studies, 40 (6).

MEYER, J.-B., 2008, « La circulation des compétences ; un enjeu pour le développement », Annuaire suisse de politique de développement, 27 (2) : 53-69.

MÜLLER, B., 2009, «Comment rendre technique un débat politique. Controverses autour des biotechnologies agricoles au sein de la FAO », Tsantsa (Revue de la Société Suisse d'ethnologie), $14: 27-36$

NATIONS UNIES, 2002, Lettre datée du 21 novembre 2002, adressée au Président de l'Assemblée générale par les représentants permanents de la Norvège et de la Suisse auprès de l'Organisation des Nations Unies, New York, Assemblée générale de l'ONU, document A/57/693.

NATIONS UNIES, 2006, Migrations internationales et développement, New York, Assemblée générale de l'ONU, document A/60/871.

ORGANISATION INTERNATIONALE POUR LES MIGRATIONS (OIM), 2010, État de la migration dans le monde 2010. L'avenir des migrations : Renforcer les capacités face aux changements, Genève, OIM.

ORGANISATION INTERNATIONALE DU TRAVAIL (OIT), 2006, Cadre multilatéral de l'OIT pour les migrations de main-d'œuvre, Genève, OIT.

ORGANISATION MONDIALE DU COMMERCE (OMC), 2004, Rapport sur le commerce mondial 2004, Genève, OMC. 
ORGANISATION MONDIALE DE LA SANTÉ (OMS), 2006, Rapport sur la santé dans le monde 2006, Genève, OMS.

ORWELL, G., 1950-1984, Paris, Gallimard.

OXFAM, 2007, Paying for People. Financing the Skilled Workers Needed to Deliver Health and Education Services for All, London, Oxfam Briefing Paper.

PHYSICIANS FOR HUMAN RIGHTS, 2004, An Action Plan to Prevent Brain Drain : Building Equitable Health Systems in Africa, Boston, Physicians for Human Rights.

PROGRAMME DES NATIONS UNIES POUR LE DÉVELOPPEMENT (PNUD), 2009, Rapport sur le développement humain 2009. Lever les barrières. Mobilité et développement humains, New York, PNUD.

RIST, G., 2002, « Le texte pris aux mots », in G. RIST (dir.), Les mots du pouvoir. Sens et non-sens de la rhétorique internationale, Paris-Genève, PUF : 25-41.

ROSENAU, J. N. \& CZEMPIEL, E., 1992, Governance without Government : Order and Change in World Politics , Cambridge, Cambridge University Press.

ROSENTAL, P.-A., 2006, « Géopolitique et État-providence. Le BIT et la politique mondiale des migrations dans l'entre-deux-guerres ", Annales HSS, 61 (1) : 99-134.

SEN, A., 1999, Development as Freedom, Oxford, Oxford University Press.

STARK, O., 1991, The Migration of Labor, Oxford, Blackwell.

UTTING, P. (ED.), 2006, « Introduction », in P. UTTING (ed.), Reclaiming Development Agendas.

Knowledge, Power and International Policy-Making, Basingstoke, Palgrave-UNRISD : 1-23.

WALLERSTEIN, I., 2006, Comprendre le monde. Introduction à l'analyse des système-monde, Paris, La Découverte.

WiCKRAMASEKARA, P., 2011, Circular Migration : a Triple Win or a Dead End, Genève, International Labour Office-Global Union Research Network.

\section{NOTES}

1. Citons en particulier l'Organisation internationale du travail (OIT) et le Haut commissariat aux droits de l'homme ( $\mathrm{HCDH})$ pour les questions de droits humains, et l'Office des Nations Unies contre la drogue et le crime (UNODC) pour ce qui concerne la « traite » et le « trafic ».

2. Cette taxe, proposée par l'économiste Jagdish bHAGWATi (BhaGWATI \& Dellafar 1973), serait prélevée sur les revenus des migrants qualifiés et reversée à leur pays d'origine, afin de compenser les pertes engendrées par leur absence et de "rembourser » les investissements publics dont ils ont bénéficiés avant leur émigration, en particulier en ce qui concerne l'éducation qu'ils ont reçue.

3. Citons par exemple le Code of Practice for NHS Employers Involved in the Recruitment of Healthcare Professionals au Royaume-Uni (2001) et le Code of Practice for the International Recruitment of Health Workers du Commonwealth (2003).

4. "Critics of the "brain drain-brain gain" debate point out that it ignores real-world patterns of international migration. Skilled workers do not "drain away" as much as "circulate" among countries in the world economy» (BANQUE MONDIALE 2009 : 168). 
5. "Governments may [...] be concerned [...] if the state has financed expensive tertiary education, only to see students leave after graduation. Where higher education is mostly financed privately, [...] this is not as much of a concern » (BANQUE MONDIALE $2007: 192$ ).

6. L'OMS (2006: 102) écrit aussi que «les enseignements tirés des efforts déployés de longue date pour améliorer la dotation des zones rurales en personnel révèlent que la formation d'agents locaux - en langue locale et en leur apprenant des savoir-faire adaptés à la situation du lieu - contribue à endiguer les départs d'agents de santé. Ce type de formation conduit souvent à des qualifications professionnelles qui ne sont pas reconnues au plan international, ce qui contribue encore à limiter les migrations ».

\section{RÉSUMÉS}

Les migrations internationales font l'objet, depuis les années 2000, d'une attention accrue de la part des organisations internationales. Cela a débouché, entre autres, sur la publication de rapports et l'émergence d'un discours international sur les migrations, qui aspirent à la fois à comprendre les enjeux migratoires et à proposer des pistes d'action politiques afin de construire des mécanismes de gouvernance internationale. De tels récits supposent cependant de surmonter les divergences d'intérêts entre les acteurs concernés (États, ONG, secteur privé notamment). Cet article analyse la manière dont ces rapports abordent la question des migrations qualifiées, lesquelles se caractérisent par des dilemmes économiques, moraux et politiques particulièrement clairs. Il montre comment les organisations internationales tentent de concilier des positions potentiellement incompatibles afin de surmonter discursivement ces dilemmes et de créer un consensus. Bien que cela fasse partie de leur mandat, le résultat de cette approche est une dépolitisation des questions liées aux migrations.

An Analysis of International Narratives on the "Brain Drain"

Migration-related issues have, since approximately 2000, been the object of increased attention at the international level. This has led, among other things, to the production of international narratives, which aim both at understanding migration and at proposing policy recommendations on how to address it, with the objective of improving the governance of migration at the global level. But this implies overcoming dilemmas stemming from the diverging interests of states and other actors (such as NGOs and the private sector). This article examines the way in which international migration narratives address skilled migration, which is characterized by some of the clearest political trade-offs between stakeholders. It argues that these narratives attempt to speak to all parties and conciliate contradictory arguments about what should be done to discursively overcome policy dilemmas and create a consensus. Although this is line with the mandate of international organizations, it depoliticizes migration issues. 
INDEX

Mots-clés : développement, discours, fuite des cerveaux, migration qualifiée, organisations internationales

Keywords : Development, Discourse, Brain Drain, Skilled Migration, International Organisations

\section{AUTEURS}

ANTONINA LEVATINO

Université Pompeu Fabra, Barcelone

\section{ANTOINE PÉCOUD}

Université de Paris 13, Sorbonne Paris Cité, Paris 